\title{
Cayley graphs as models of deterministic small-world networks
}

\author{
Wenjun Xiao a ${ }^{\text {, Behrooz Parhami }}{ }^{\text {b,* }}$ \\ a Department of Computer Science, South China University of Technology, Guangzhou 510641, P.R. China \\ ${ }^{\mathrm{b}}$ Department of Electrical \& Computer Engineering, University of California, Santa Barbara, CA 93106-9560, USA
}

Received 6 May 2004; received in revised form 30 May 2005

Available online 28 October 2005

Communicated by F. Dehne

\begin{abstract}
Many real networks, including those in social, technological, and biological realms, are small-world networks. The two distinguishing characteristics of small-world networks are high local clustering and small average internode distance. A great deal of previous research on small-world networks has been based on probabilistic methods, with a rather small number of researchers advocating deterministic models. In this paper, we further the study of deterministic small-world networks and show that Cayley graphs may be good models for such networks. Small-world networks based on Cayley graphs possess simple structures and significant adaptability. The Cayley-graph model has pedagogical value and can also be used for designing and analyzing communication and the other real networks.
\end{abstract}

(C) 2005 Elsevier B.V. All rights reserved.

Keywords: Average internode distance; Cayley graph; Clustering coefficient; Interconnection network; Low-diameter network

\section{Introduction}

In the past few years, an array of discoveries have added to our understanding of complex networks [2]. Many real networks, including those in social, technological, and biological realms, are small-world networks. As graphs, such networks have clustering coefficients much larger than those of random networks, while their average internode distances are comparably small (growing as the logarithm of the number $n$ of nodes). In 1998, Watts and Strogatz [9] described stochastic models of small-world networks. Their method is to begin with a structured network such as a loop net-

\footnotetext{
* Corresponding author. Tel./fax: +1 805893 3211/3262.

E-mail addresses: wjxiao@scut.edu.cn (W. Xiao), parhami@ece.ucsb.edu (B. Parhami).
}

work, which has high clustering but also a large average internode distance, and to randomly rewire the edges so as to reduce the average internode distance. Their models, dubbed "small-world networks" (corresponding to the popular notion of "six degrees of separation") offer high clustering, like loop networks, yet possess small average internode distances, as in random networks.

Subsequently, Comellas and Sampels [6] discovered that constant-degree deterministic small-world networks are also feasible. One motivation in introducing deterministic models for small-world networks is to facilitate the understanding of their behavior. This approach also permits a direct calculation of relevant network parameters and thus allows the design of specific small-world networks for applications such as new communication systems and computer architectures. 
In this paper we show that Cayley graphs are excellent models for small-world networks, in the sense that with suitable choice of relevant parameters, they can be adapted to possess the distinguishing characteristics of such networks. Our models offer simple structures as well as significant adaptability. The Cayley-graph model has pedagogical value and can also be used for designing and analyzing communication and the other real networks.

\section{Cayley-graph network models}

It is known that Cayley graphs are excellent models for interconnection networks [1,3]. Many well-known and practically useful interconnection networks are Cayley graphs. For example, hypercube and cube-connected cycles networks are Cayley graphs. We shall demonstrate that Cayley graphs can also serve as excellent models for small-world networks.

We first introduce some notation and technical terms. A digraph $\Gamma=(V, E)$ is defined by a set $V$ of vertices and a set $E$ of arcs or directed edges. The set $E$ is a subset of elements $(u, v)$ of $V \times V$. If the subset $E$ is symmetric, that is, $(u, v) \in E$ implies $(v, u) \in E$, we identify two opposite $\operatorname{arcs}(u, v)$ and $(v, u)$ by the undirected edge $(u, v)$. We then have a graph. Let $G$ be a finite group and $S$ a subset of $G$ (we do not deal with infinite groups in this paper). The subset $S$ is said to be a generating set for $G$, and the elements of $S$ are called generators of $G$, if every element of $G$ can be expressed as a finite product of the powers of the elements in $S$. In this case, we also say that $G$ is generated by $S$.

The Cayley digraph of a group $G$ and the subset $S$ of $G$, denoted by $\operatorname{Cay}(G, S)$, has vertices that are elements of $G$ and arcs that are ordered pairs $(g, g s)$ for $g \in G, s \in S$. If $S$ is a generating set of $G$, then we say that $\operatorname{Cay}(G, S)$ is the Cayley digraph of $G$ generated by $S$. If $1 \notin S(1$ is the identity element of $G)$ and $S=S^{-1}$, then $\operatorname{Cay}(G, S)$ is a simple (undirected) graph. For more definitions and basic results on graphs and groups we refer the reader to [5], for instance, and on interconnection networks to $[7,8]$. Unless noted otherwise, all graphs in this paper are undirected graphs.

\section{Clustering coefficient}

Let $G$ be a finite group. Assume that $\Gamma=\operatorname{Cay}(G, S)$ for some generating set $S$ of $G$, where $1 \notin S, S=S^{-1}$. Then, $\Gamma$ is a Cayley graph of constant degree $d=|S|$, every node $v$ of $\Gamma$ has exactly $d$ neighbors, and at most $d(d-1) / 2$ edges exist between these $d$ neighbors of $v$. The fraction $C_{v}$ of these allowable edges that actually exist between the neighbors is known as the clustering coefficient of node $v$. The average of $C_{v}$ over all $v \in G$ is the clustering coefficient $C$ of the graph $\Gamma$.

Because $\Gamma$ is symmetric, the clustering coefficient of every node is the same $\left(C_{v}=C\right)$. Thus, we only need to consider the clustering coefficient of node 1 , the identity element of $G$. The set of neighbors of node 1 is $S$. If $s_{1}, s_{2} \in S$, then $s_{1}$ and $s_{2}$ are adjacent if and only if there is $s \in S$ such that $s_{2}=s_{1} s$. Assume that $H \subseteq S$ and $H \cup\{1\}$ is a subgroup of $G$. Then $s_{1} s_{2} \in H$ when $s_{1}, s_{2} \in H$. Hence, there are at least $|H|(|H|-1) / 2$ edges among the set $S$ of neighbors of node 1 . As a result, the clustering coefficient of $\Gamma$ will be large if $H$ can be chosen to be large. This is the central idea of our method.

In contrast to the method of [9], we start from networks with small clustering coefficients and small average internode distances and proceed to increase the clustering coefficient by means of adding elements of $G$ into $S$, while ensuring that the average internode distance remains small. We present the essence of our method via two examples.

\section{First example network}

Let $G_{1}=Z_{2}^{t}$ be an elementary commutative group of order $2^{t}$. Consider an integer $l$ satisfying $1 \leqslant l \leqslant t$. Define $H_{1}=\left\{\left(x_{1}, x_{2}, \ldots, x_{l}, 0^{t-l}\right) \mid\left(x_{1}, x_{2}, \ldots, x_{l}\right) \in\right.$ $\left.Z_{2}^{l}\right\} \backslash\{(0,0, \ldots, 0)\}$, where $(0,0, \ldots, 0)$ is the identity element of $G_{1}$, and $S_{1}=H_{1} \cup\left\{\left(x_{1}, x_{2}, \ldots, x_{t}\right) \in\right.$ $Z_{2}^{t} \mid$ only one of $x_{1}, x_{2}, \ldots, x_{t}$ is 1$\}$. Assume that $\Gamma_{1}=$ $\operatorname{Cay}\left(G_{1}, S_{1}\right)$. When $l=1$, the network $\Gamma_{1}$ is the wellknown hypercube (binary $t$-cube) network; it becomes the complete graph for $l=t$. One can easily verify that $\left|H_{1}\right|=2^{l}-1$ and $\left|S_{1}\right|=\left|H_{1}\right|+t-l=2^{l}+t-l-1$. Hence, we have established the following result.

\section{Proposition 1. The clustering coefficient of $\Gamma_{1}$ is}

$$
C_{1}=\frac{\left(2^{l}-1\right)\left(2^{l}-2\right)}{\left(2^{l}+t-l-1\right)\left(2^{l}+t-l-2\right)} .
$$

Let the diameter (maximum distance between any pair of nodes) of $\Gamma_{1}$ be $D\left(\Gamma_{1}\right)$ and $n=2^{t}$. Then $\left|G_{1}\right|=$ $n$ and $D\left(\Gamma_{1}\right) \leqslant t=\log _{2} n$. Thus the average internode distance of $\Gamma_{1}$ is no greater than $\log _{2} n$. Based on the results above, we can choose the value of $l$ such that the clustering coefficient $C_{1}$ is large and the degree of $\Gamma_{1}$ is still small. For instance, with $l=\log _{2} t$, or equivalently, $t=2^{l}$, the clustering coefficient is:

$C_{1}=\frac{(t-1)(t-2)}{\left(2 t-\log _{2} t-1\right)\left(2 t-\log _{2} t-2\right)}$. 
Therefore $C_{1} \rightarrow \frac{1}{4}$ when $t \rightarrow \infty$. More generally, let $a=\left(2^{l}-1\right) / t$. Then we have:

$C_{1}=\frac{a t(a t-1)}{(a t+t-l)(a t+t-l-1)}$.

In this case, $C_{1} \rightarrow a^{2} /(a+1)^{2}$ when $t \rightarrow \infty$. By suitably choosing $a$, we can obtain different clustering coefficients for $\Gamma_{1}$, while maintaining a small node degree equal to $a t+t-l$.

One can choose $S_{1}$ and $H_{1}$ as subsets of $G_{1}$ in many different ways, leading to diverse small-world network models. However, the degree of $\Gamma_{1}$ is at least equal to $t=\log _{2} n$. We consider Cayley graphs of constant degrees in the following example.

\section{Second example network}

Let $N=Z_{2}^{q}$, where $q \geqslant 3$. Suppose that $K=Z_{q}$ is a cyclic group of order $q$ and $G_{2}=Z_{2} w r Z_{q}$ is a semidirect product of $N$ by $K$. Consider integers $l$ and $t$ satisfying $1 \leqslant l \leqslant t<q$ and let $S_{2}=H_{2} \cup$ $\left\{\left(0^{q}, 1\right),\left(0^{q},-1\right)\right\} \cup\left\{\left(x_{1}, x_{2}, \ldots, x_{t}, 0^{q-t+1}\right) \mid\left(x_{1}, x_{2}\right.\right.$, $\left.\ldots, x_{t}\right) \in Z_{2}^{t}$, and only one of $x_{1}, x_{2}, \ldots, x_{t}$ is 1$\}$, where $H_{2}=Z_{2}^{l} \backslash\{(0,0, \ldots, 0)\}$ and $(0,0, \ldots, 0)$ is the identity element of $G_{2}$. Let $\Gamma_{2}=\operatorname{Cay}\left(G_{2}, S_{2}\right)$. When $t=l=1$, the network $\Gamma_{2}$ is the well-known cube-connected cycles network. One can easily verify that $\left|H_{2}\right|=2^{l}-1$ and $\left|S_{2}\right|=\left|H_{2}\right|+t-l+2=2^{l}+t-l+1$. Thus, we have established the following result.

Proposition 2. The clustering coefficient of $\Gamma_{2}$ is

$C_{2}=\frac{\left(2^{l}-1\right)\left(2^{l}-2\right)}{\left(2^{l}+t-l+1\right)\left(2^{l}+t-l\right)}$.

We know that the degree of $\Gamma_{2}$ is $\left|S_{2}\right|=2^{l}+t-l+1$ and the order of $G_{2}$ is $\left|G_{2}\right|=2^{q} q$. Hence the degree of $\Gamma_{2}$ does not depend on the order of $G$ and is a constant when $t$ and $l$ are fixed. The diameter of $\Gamma_{2}$ is no greater than that of the cube-connected cycles of the same order, which is in turn at most $5 q / 2-1$. Thus, the graph $\Gamma_{2}$ is a small-world network model. By suitably choosing $t$ and $l$, we can get different clustering coefficients which are large.

\section{Discussion}

We have established that Cayley graphs are indeed good models for small-world networks. Our models are structurally simpler, as well as more amenable to theoretical development and comparative evaluation, than those in [6] or [9]. Unlike the probabilistic models of
[9], our networks are characterized by closed-form, exact formulas for various properties, which lead to intuitive and fairly precise mechanisms for varying the pertinent parameters. The deterministic models of [6], which borrow from techniques previously found useful for constructing large, low-diameter networks [4], do not offer the same simplicity and precision. In particular, the method of starting with a regular degree- $d$ network and replacing each of its nodes with the $d$-node complete graph $K_{d}$, which is the only node-symmetric construction proposed in [6], yields only one smallworld network for each starting configuration. Our approach, on the other hand, results in a family of networks which offer a range of parameters.

We believe that our models will have important applications to diverse research fields, including in parallel architectures and communication networks. By suitably choosing the parameters of the Cayley-graph models, they can be made to mimic many real networks of the types found in social, technological, and biological domains. This constitutes a fertile field for further investigation.

\section{Acknowledgements}

Research of W. Xiao was supported by the Natural Science Foundation of China and Guangdong Province. The authors are grateful to an anonymous referee for valuable suggestions.

\section{References}

[1] S.B. Akers, B. Krishnamurthy, A group theoretic model for symmetric interconnection networks, IEEE Trans. Comput. 38 (1989) 555-566.

[2] R. Albert, A.L. Barabasi, Statistical mechanics of complex networks, Rev. Modern Phys. 74 (2002) 47-97.

[3] F. Annexstein, M. Baumslag, A.L. Rosenberg, Group action graphs and parallel architectures, SIAM J. Comput. 19 (1990) 544-569.

[4] J.-C. Bermond, C. Delorme, J.-J. Quisquater, Tables of large graphs with given degree and diameter, Inform. Process. Lett. 15 (1) (1982) 10-13.

[5] N. Biggs, Algebraic Graph Theory, Cambridge University Press, Cambridge, 1993.

[6] F. Comellas, M. Sampels, Deterministic small-world networks, Physica A 309 (2002) 231-235.

[7] F.T. Leighton, Introduction to Parallel Algorithms and Architectures: Arrays, Trees, Hypercubes, Morgan Kaufmann, Los Altos, CA, 1992.

[8] B. Parhami, Introduction to Parallel Processing: Algorithms and Architectures, Plenum, New York, 1999.

[9] D.J. Watts, S.H. Strogatz, Collective dynamics of 'small-world' networks, Nature 393 (1998) 440-442. 